\title{
A principled stance
}

\section{Martyn Poliakoff and Paul Anastas}

$n$ Chinese, chemistry is the 'mixing science', whereas in Dutch, it's the 'separation art'. But whatever their nationality, academic chemists often do not know in detail how the chemicals they use are made, or how their chemistry affects the biosphere. They view industrial chemistry as meat-eaters view the slaughterhouse - the gory details are glossed over and the fate of the waste is ignored. On the other hand, much of the general public recoils at the mere mention of 'chemicals', yet we are all clothed, fed, washed and transported by the products of the chemical and pharmaceutical industries. While we benefit from increased quality and length of life thanks to chemicals, the industry that makes them is frequently accused of degrading the Earth.

Society relies on chemicals. Like a good cook, the industrial chemist recycles waste. Yet many types of waste are dangerous, leaving no option but costly disposal. With the global population rising and standards of living increasing, current methods of chemical production are unsustainable. As production rises to meet demand, waste levels will soar and landfill sites will be exhausted. Manufacturers will be increasingly restricted by environmental legislation; enforcement agencies will become increasingly overloaded; and costs of waste treatment will stifle innovation.

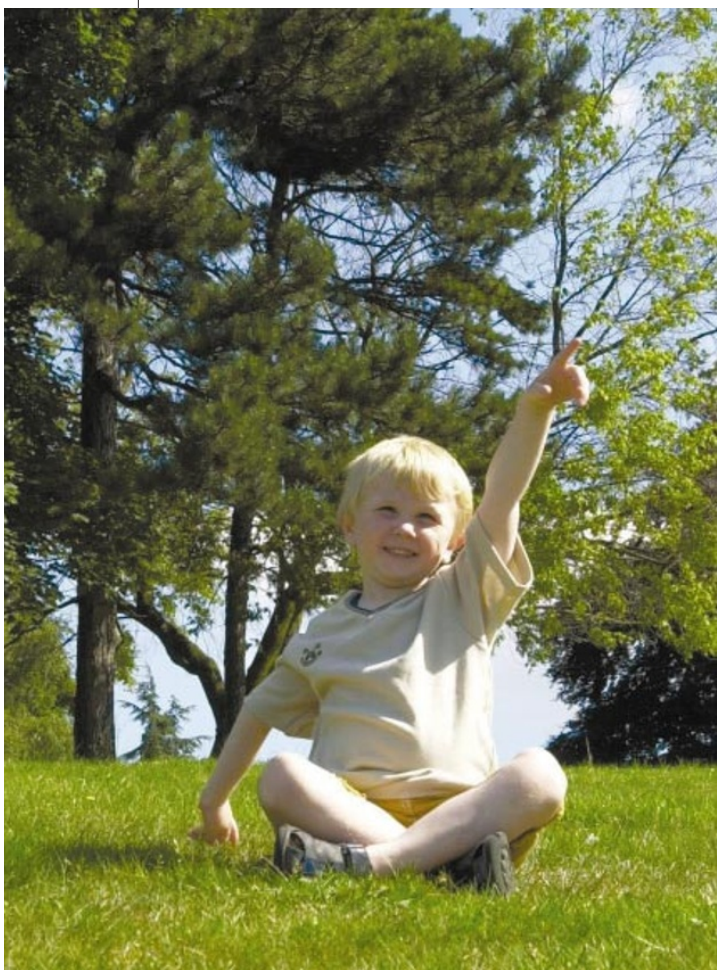

Green future: the world needs cleaner chemistry.
A new approach is needed to remould attitudes constructively, and to attract young people to the field of chemistry. Step forward 'green chemistry', which aims to create products that are as harmless as possible and therefore require less regulation. The concept is enshrined in a set of 12 principles, ranging from minimizing waste to avoiding accidents. Individually, these principles are not new; what is potentially revolutionary is grouping them together so that chemists can focus on using classical chemistry to design products with less environmental impact. For example, non-toxic carbon dioxide is now used to make lighter, stronger building materials and to replace chlorinated organic solvents in chemical reactions and dry-cleaning. Integrating energy, waste and toxicity issues when thinking about new processes and reactions has proved to be very effective.

Although some industrial processes are efficient, others are extremely wasteful, requiring costly handling and disposal of chemicals. The more stages a chemical process involves, the more potential it has for creating waste. So green chemistry inspires a kind of chemical 'golf match', in which fewer steps represent a better environmental 'score' and the best result is, of course, a hole in one. For example, a new process for producing the anti-inflammatory drug ibuprofen halves the number of steps, makes them all catalytic and more than doubles the atom efficiency of the process. Green chemistry not only leads to cleaner and more efficient processes, but can increase profitability by eliminating many of the traditional costs of treatment, disposal, liability and regulatory compliance.

Green chemistry involves more than just tidying up existing processes - if it were that simple, process economics would already be driving manufacturers in that direction. Rather, it identifies a need to design new, safer molecules. Often, the toxicity and the usefulness of a compound arise from different parts of the molecule; for example, a couple of strategically placed methyl groups added to a dye molecule makes it much less toxic but leaves its dyeing properties intact. Similar minor structural modifications can increase the biodegradability of molecules and reduce their environmental impact. Linking structure and toxicity gives synthetic chemists a new perspective, allowing them to design new pesticides that are toxic only to target organisms, with a fraction of the usual dose, and which do not persist in the environment.

Green chemistry has captured the imagination of many chemists. But some industrial chemists believe that their very successful efforts at cleaning up have been overlooked, and some academics fear that green chemistry

\section{Green chemistry}

Already, young people are beginning to perceive chemistry as a key to saving our environment rather than a tool for its destruction.

is 'soft' science. Although understandable in historical contexts, neither view is correct. Green chemistry is a new partnership, bringing together the efforts of industrial and academic research and building rapidly on the past successes of both. New solvents, feedstocks, catalysts and processes, producing everything from new, biologically derived, renewable plastics to lead-free automotive paint, are being developed throughout the world.

Many challenges remain. How can chemical production shift from unsustainable petroleum feedstocks to renewable biomass? Can some of the vast quantities of carbon dioxide vented into the atmosphere be converted into useful chemicals? Can the environmental impact of agrochemicals be reduced while increasing food production? Green chemistry will attract the new generation of chemists needed to solve these problems. Already, young people are beginning to see chemistry as a key to saving our environment rather than a tool for its destruction.

Will green chemistry take over all of chemistry? Far from it - the revolution of one generation becomes the orthodoxy of the next. The 12 principles are so obvious that chemists of the future will wonder why it took so long to integrate them into the mainstream. After all, why make chemicals wastefully and expensively, when they can be produced cheaply and cleanly?

Martyn Poliakoff is a research professor and Paul

Anastas is a special professor at the School of Chemistry, University of Nottingham,

University Park, Nottingham NG7 2RD, UK.

\section{FURTHER READING}

Anastas, P. \& Warner, J. Green Chemistry: Theory and Practice (Oxford Univ. Press, London, 1998).

Anastas, P. \& Lankey, R. Chem. Britain 37, 26-29 (2001). Hamley, P. A. \& Poliakoff, M. Chem. Engineer72, 24-27 (2001).

Ritter, S. K. Chem. Eng. News 79, 24-37 (2001).

\section{WEBLINKS}

Green chemistry network:

http://www.rsc.org/networks/gen

What is green chemistry?:

http://www.epa.gov/opptintr/greenchemistry/ whatis.htm

Green chemistry at Nottingham:

http://www.nottingham.ac.uk/greenchemistry 